\title{
Los beneficios de la participación en múltiples deportes para los tenistas
} jóvenes

\author{
E. Paul Roetert, Ronald B. Woods y Neeru A. Jayanthi
}

\section{RESUMEN}

Durante la última década, el tema de la especialización deportiva temprana ha sido ampliamente debatido. En este artículo revisamos algunas de las investigaciones recientes relacionadas con el tenis y proporcionamos algunas recomendaciones para la participación en múltiples deportes con el fin de ayudar a reducir las lesiones, mejorar las habilidades deportivas generales y permitir el disfrute de una participación física para toda la vida. Además, compartimos nuestros objetivos para los jugadores de tenis jóvenes de todos los niveles y ofrecemos consejos de entrenamiento específicos.
Palabras clave: Participación deportiva, muestreo deportivo, especialización deportiva temprana

Recibido: 25 Junio 2018

Aceptado: 10 Julio 2018

Autor correspondiente:

E. Paul Roetert.

Correo electrónico: eproetert@gmail.com

\section{INTRODUCCIÓN}

Durante las últimas décadas, hemos tenido la oportunidad de viajar por el mundo realizando diferentes actividades relacionadas con el negocio del tenis. Estas actividades incluyen participar en las áreas de entrenamiento, formación de entrenadores, ciencias y medicina del deporte y colaborar en varios roles administrativos. A través de estos roles, hemos llegado a amar y comprender el deporte desde diferentes perspectivas y a disfrutar sus muchos beneficios, deseando compartir esta pasión alrededor del mundo. Los entrenadores cuentan con esta misma oportunidad, particularmente en lo que concierne a la introducción de los niños menores al deporte del tenis y a la enseñanza de las habilidades necesarias para disfrutar de un juego para toda la vida. Juntos, y es de esperar, con la ayuda y soporte de la ITF, nuestro interés se focalizará en el logro de los siguientes seis objetivos:

- Atraer a los niños al deporte, y en nuestro caso, al tenis, específicamente.

- Retener a los niños en el tenis el mayor tiempo posible.

- Maximizar el desarrollo de talentos al más alto nivel buscado y mostrar su habilidad para lograrlo.
- Establecer las bases para toda una vida de actividad física, promocionando y guiando a los niños hacia un estilo de vida alfabetizado físicamente.

- Proporcionar un entrenamiento libre de lesiones y un entorno competitivo.

- Introducir a los niños a una variedad de deportes y actividades físicas, comenzando desde temprana edad.

Los entrenadores, ciertamente, pueden jugar un rol significativo a la hora de lograr esas metas, proporcionando un ambiente saludable, positivo, y de formación conducente al desarrollo de habilidades apropiadas; mientras los formadores de entrenadores y los investigadores del tenis basan su esfuerzo de la siguiente manera: 1.) proporcionando de manera continua un mayor conocimiento en áreas relacionadas con la formación de entrenadores, 2.) Compartiendo las aplicaciones prácticas de modo amigable, en base a una investigación sólida y a las mejores prácticas, y 3.) Desarrollando un conjunto de conocimientos adecuado para la edad, para todos los formadores de entrenadores de todos los niveles (Roetert y Bales, 2014).

Un área de preocupación que cada uno de estos grupos (entrenadores, formadores de entrenadores, e investigadores/ 
científicos del tenis) ha estado tratando particularmente durante la última década es la tendencia hacia "la especialización en el deporte único" en edades tempranas, frecuentemente basada

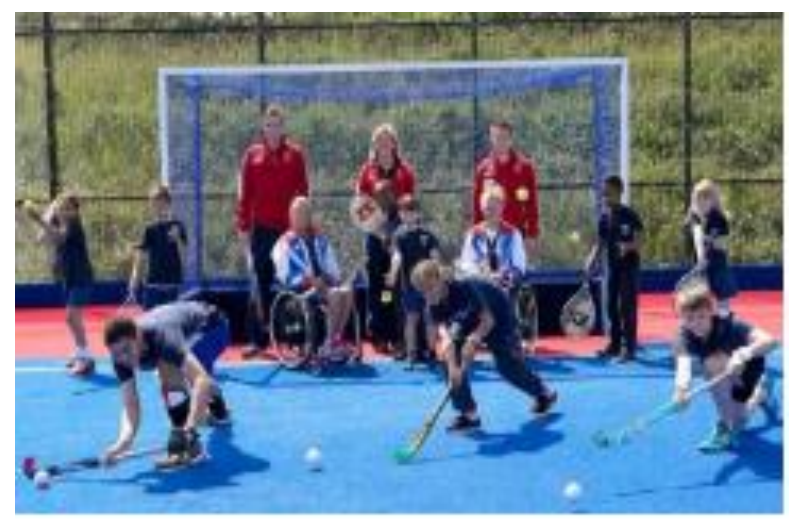

en la guía de entrenadores o padres, quienes desean maximizar el potencial de los niños para que se transformen en atletas de elite. Lamentablemente, los resultados de este enfoque pueden potencialmente conducir a una carrera deportiva corta, o quizás cualquier otra actividad física, debido a lesiones por sobreuso físico o agotamiento psicológico. Gould y colegas $(1996,1997)$ estudiaron el agotamiento (burnout) en jóvenes tenistas y concluyeron que sus causas son la combinación e interacción de cuatro factores; lesión física o fatiga, preocupaciones logísticas y exigencias de tiempo, exigencias sociales de familiares y pares, preocupación psicológica, como la presión por los resultados de la competición y la clasificación. A menudo, se gasta mucho dinero, tiempo y esfuerzo para lograr el éxito en los más altos niveles, aún sin la presencia de talento o habilidades atléticas, dejando potencialmente a los jugadores con posibilidades limitadas de realizar otras actividades físicas para toda la vida.

En qué medida los jóvenes atletas necesitan especializarse a temprana edad es uno de los debates más inspiradores y pertinentes del deporte juvenil de hoy, y los investigadores de todo el mundo discuten el tema desde hace décadas (Horton, 2012). Sin embargo, en base a la investigación reciente, creemos que la estrategia del "muestreo deportivo" tiene muchas ventajas, en particular, (pero no exclusivamente) en lo que concierne a los grupos de menor edad. De hecho, el muestreo deportivo temprano y la diversificación no parecen impedir el éxito cuanto se alcanza el pico de rendimiento después de la madurez total. Además, la diversificación en edades tempranas parece vincularse positivamente con una carrera deportiva más larga, una vida de actividad física y una mejor salud y bienestar general. (LaPrade y cols, 2016, Coté y cols. 2009). Al mismo tiempo, hay ciertos deportes, incluyendo la gimnasia femenina, el patinaje artístico, el buceo y la danza que requieren una especialización temprana (antes de los 12 años), pues el rendimiento pico generalmente ocurre durante la adolescencia media o tardía, antes de la madurez total. La especialización temprana en estos deportes ha demostrado ser un vaticinador de éxito en los niveles elite. ( Coté y cols. 2009).

\section{REDUCIR LAS LESIONES}

Revisando la literatura, vemos que los atletas de deportes individuales como el tenis son más proclives a especializarse en un sólo deporte que los atletas de deportes por equipo También, hay una clara evidencia de que hay un mayor riesgo de lesiones por sobre-uso entre los deportistas jóvenes que participan en un entrenamiento especializado de un deporte único (LaPrade y cols., 2016, Jayanthi y cols., 2015, Goodway \& Robinson, 2015).

Probablemente, una de las razones principales sea que los atletas especializados en un deporte único cuentan con mayores volúmenes de entrenamiento (Pasulka, 2017), aunque varios otros estudios ya mencionados se ajustaron por edad y volumen de entrenamiento. Por lo tanto, podría haber aún un riesgo independiente para la especialización deportiva. Aunque el entrenamiento temprano especializado en deportes individuales como el tenis ha demostrado que conduce a riesgos tanto de lesiones por sobre-uso como por agotamiento en los jugadores adolescentes, la mayor parte de esa investigación se ha focalizado en los atletas jóvenes varones. No obstante, un estudio reciente de Jayanthi \& Dugas (2017) destaca la evidencia emergente de patrones similares basados en la especialización deportiva temprana también en atletas adolescentes mujeres.

Como entrenadores de tenis, ustedes y los padres, necesitan recordar el volumen total de tenis llevando registros precisos del tiempo de entrenamiento de cada jugador, la intensidad (juego de partidos vs práctica) y frecuencia diaria, semanal y mensual). Al mismo tiempo, si bien no existe la fórmula mágica perfecta para el entrenamiento de tenis óptimo, se debe tener en cuenta el nivel de motivación intrínseca, el nivel de energía gastado y el aparente disfrute del jugador, determinando estos factores por medio de la observación, comentarios extraídos de los jugadores y la evaluación de los padres. Dejar por lo menos una hora al mes para revisar estos datos junto con el jugador y sus padres, y acordar juntos un plan de entrenamiento para las futuras semanas. Además, se debe monitorear a los jugadores cuya horas de práctica por semana superen su edad, más de 16 horas por semana en entrenamiento intensivo, y quienes se especialicen solamente en tenis, para controlar los indicadores de agotamiento o disminuciones potenciales de rendimiento debido al entrenamiento excesivo (Jayanthi y cols., 2011 y 2015). Según una investigación de Jayanthi y cols. (2011 y 2015), el entrenamiento de tenis cada semana debe estar ligado a la edad cronológica relacionada con las horas de entrenamiento. Esta regla general reducirá las probabilidades de lesiones por sobreuso y el subsiguiente tiempo fuera de entrenamiento. 


\section{Consejo de entrenamiento 1}

Los tenistas jóvenes deben seguir la regla de edad versus las horas: Entrenar por semana no una mayor cantidad de horas que la edad real. Por ejemplo, un jugador de 12 años debe limitar su entrenamiento de tenis a 12 horas por semana.

\section{Consejo de entrenamiento 2}

Además de ajustar y moderar la frecuencia, volumen e intensidad de entrenamiento en base a la edad del jugador $y$ al momento de la temporada, se debe enfatizar la solidez del golpe, técnica de movimiento para ayudar a mitigar el riesgo de lesiones. Un ejemplo de cómo limitar la intensidad del entrenamiento consiste en limitar el número de torneos/ partidos dentro de un año y dentro de cada mes, para permitir el "tiempo de recuperación" suficiente, no sólo físico sino también emocional y psicológico. Otro factor clave es programar deliberadamente varias semanas sin entrenamiento de tenis, periódicamente, cada año.

\section{MEJORAR LAS HABILIDADES DEPORTIVAS}

La expresión "destrezas motrices fundamentales" se viene utilizando desde hace años, particularmente en relación con la enseñanza a los jóvenes de las habilidades físicas para tener éxito durante toda la vida. Un estudio reciente sugiere reemplazar esa frase por "habilidades fundacionales de movimiento" para un mejor reconocimiento de las actividades físicas, a lo largo de la vida, el potencial cultural y las variaciones geográficas, así como también las sinergias entre los factores físicos y psicológicos: Hulteen y cols. (2018) proponen este cambio para ayudar a avanzar con la aplicación de principios de desarrollo motriz dentro del dominio de la salud pública. Como ejemplo, los movimientos para el entrenamiento de la resistencia, los golpes de natación y andar en bicicleta. Esta idea parece estar muy en línea con el concepto de "Alfabetización Física" que se debatió en la edición de agosto de 2016 de la Revista de Entrenamiento y Ciencias del Deporte de la ITF (Roetert y cols., 2016). La Asociación de Alfabetismo Físico define este Alfabetismo Físico como la motivación, confianza, competencia física, conocimiento y comprensión para valorar y asumir la responsabilidad para el compromiso en las actividades físicas para toda la vida (IPLA, 2016; Roetert, y cols., 2018). Ambos conceptos destacan el enfoque holístico hacia la actividad física (física y psicológica), reconociendo los beneficios de la participación multi-deportiva (muestreo deportivo) y un foco general en proporcionar beneficios para la salud gracias la participación, a lo largo de toda la vida.

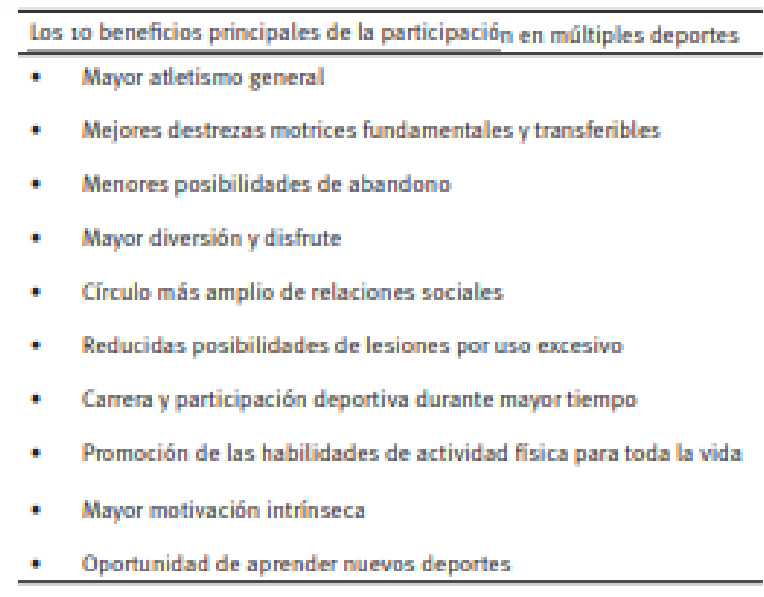

Tabla 1. Los 10 beneficios principales de la participación en mültiples deportes.

En todo nivel de rendimiento elite, el modelo recomendado es un modelo de muestreo especializado, por lo menos según se recomienda para el fútbol. Este modelo, descripto por Sieghartsleitner y cols., 2018, permite un muestreo especializado con alto grado de especificidad dentro de la participación deportiva temprana (especialización), que se enriquece con la diversidad deportiva específica que surge de un amplio rango de entornos dentro del deporte (muestreo). Ciertamente, esperamos y recomendamos, que este tipo de estudio se replique con el foco en los tenistas. Tomando consciencia de que no todos los tenistas junior serán competidores de nivel mundial, éstos son importantes conceptos que todos los entrenadores de tenis deben reconocer. Las palabras "muestreo deportivo" son apropiadas pues permiten que los deportistas se focalicen en sus deportes principales, disfrutando la participación en otros deportes y actividades. Los deportistas jóvenes que fueron introducidos al muestreo deportivo son más proclives a continuar participando en actividades sociales a medida que aumenta su edad (Gallant y cols, 2017).

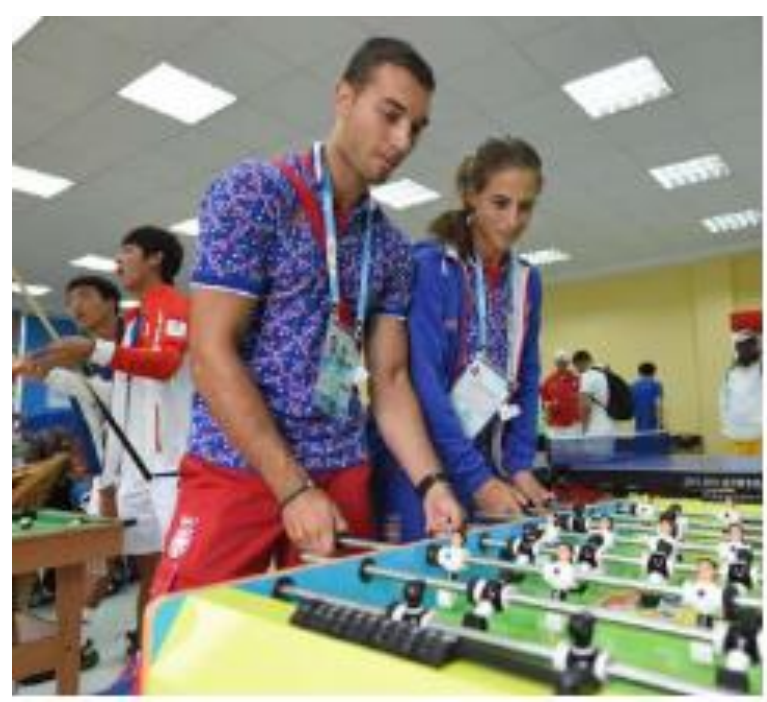


En algún momento, los atletas de elite tendrán que decidir su mejor deporte, y dedicar su energía y esfuerzo a una práctica deliberada, y jugar rutinas junto con mayores desafíos en el juego de torneos competitivos. Otros deportes y actividades físicas pueden aún jugar un rol más recreativo durante los días y períodos de recuperación y descanso del entrenamiento de tenis. El momento óptimo para la especialización deportiva parece ser entre los 12 y 15 años, con variaciones individuales según la madurez general, física, emocional, mental y social. Las mujeres suelen madurar algo más temprano que los varones, pero alrededor de los 16 años, los atletas verdaderos típicamente se sumergen en el deporte de su elección.

\section{Consejo de entrenamiento 3}

Considerar recomendar otras actividades deportivas a los jugadores para ayudar a desarrollar las habilidades atléticas fundacionales, especialmente aquéllas que puedan transferirse a habilidades tenísticas. Por ejemplo, los movimientos de fútbol, básquetbol, hockey, vóley, béisbol/softball, destrezas para el lanzamiento y captura.

\section{Consejo de entrenamiento 4}

Recomendar varias experiencias para que los niños prueben un deporte de equipo. Este enfoque amplía y apoya el atletismo general, las habilidades físicas, el sentido del juego y la aplicación de estrategias y tácticas.

\section{DISFRUTAR LA PARTICIPACIÓN DURANTE TODA LA} VIDA

El beneficio de participar en una actividad física a medida que los jugadores envejecen no debe subestimarse. El tenis es un deporte fantástico para toda la vida, y hay competiciones disponibles para muchos grupos de edades diferentes. De hecho, indica la investigación, que cuánto más favorable la experiencia deportiva para los jóvenes atletas, mayor será la posibilidad de que dichos atletas participen en actividades físicas como adultos (Miller y Siegel, 2017). Además, estos autores mencionan que los entrenadores y los padres deben focalizar en la experiencia de los deportes de juventud, independientemente y más allá de los triunfos y derrotas. Una experiencia positiva con los deportes de juventud puede durar la vida entera. Nuevamente, esto está en línea con el concepto de alfabetismo físico, que se focaliza en la actividad física como un viaje de toda la vida (Roetert y cols., 2018) así como también las "habilidades de movimiento fundacionales", que promocionan cómo varias formas de movimiento apoyan y mantienen una vida de actividad física (Hulteen, 2018).

\section{Consejo de entrenamiento 5}

Considerar por lo menos una introducción a otros deportes y habilidades que puedan ser accesibles, y prepararse para una vida de actividades como la natación, el ciclismo, el tenis, el golf, el yoga, las artes marciales y varias actividades físicas para lograr el estiramiento, la flexibilidad y la resistencia aeróbica.

Consejo de entrenamiento 6 Permitir que los jugadores busquen oportunidades de ampliar la red social, conocer nuevos entrenadores, hacer nuevas amistades con niños de otras actividades deportivas. Esto puede ayudar a evitar el agotamiento emocional-social debido a un -concepto propio y una confianza inmaduros, y a camarillas y amistades sociales dentro de la comunidad tenística.

\section{Consejo de entrenamiento 7}

Evitar el agotamiento mental debido a las mismas rutinas, la falta de variedad en la práctica, las mesetas de aprendizaje y rendimiento que pueden producir estancamiento. Permitir un tiempo para la recuperación saludable y para el descanso del deporte y de la competición.

\section{COMENTARIOS PARA RESUMIR}

La especialización deportiva se define como el entrenamiento intensivo durante todo el año, en un único deporte, excluyendo los otros (Jayanthi y cols., 2013). Los expertos en medicina/ ciencias del deporte notan que, con los años, ha habido un incremento en la especialización deportiva, lo que a su vez, probablemente incremente el riesgo de lesión y agotamiento en los jóvenes atletas. Además, las familias tienen que asignar significativos recursos financieros para apoyar estos patrones de entrenamiento especializado (Jayanthi y cols., 2018). Estas preocupaciones, de hecho, afectan el deporte de tenis $y$, creemos que los lectores de esta publicación (investigadores de tenis, formadores de entrenadores, y expertos en entrenamiento de muchos y diferentes países) pueden ayudar a lograr una diferencia, con las organizaciones y con los jugadores a quienes alcanzan, en todo el mundo. Aún se debe trabajar arduamente para maximizar las experiencias positivas para los jugadores jóvenes. Esto, ciertamente, incluye conducir, promocionar, y compartir la investigación relacionada con la especialización deportiva desde la niñez. Esperamos poder proporcionarle una actualización sobre los futuros resultados logrados en los años venideros. Mientras tanto, considere los seis objetivos a los que nos hemos referido y los consejos de entrenamiento que recomendamos para sus jugadores.

\section{REFERENCIAS}

Coté, J., Lidor, R., \& Hackfort, D. 2009. ISSP Position Stand: To Sample or to Specialize? Seven Postulates About Youth Sport Activities that Lead to Continued Participation and Elite Performance. International Society of Sports Psychology. (ISSP). https://doi.org/10.1080/1612197X.2009.9671889

Ericsson, K.A., Krampe, R.T. \& Tesch-Romer, C. (1993). The role of deliberate practice in the acquisition of expert performance. Psychological Review. 100:363-406. https://doi.org/10.1037/0033-295X.100.3.363 
Gallant, F., O'Loughlin, J.L., Brunet, J., et al. (2017). Childhood Sports Participation and Adolescent Sport Profile. Pediatrics. 140(6):e20171449. https://doi.org/10.1542/peds.2017-1449

Goodway, J.D. \& Robinson, L.E. (2015). Developmental Trajectories in Early Sport Specialization: A Case for Early Sampling from a Physical Growth and Motor Development Perspective. $\begin{array}{lllll}\text { Kinesiology } & \text { Review. } & 4 & 267 & -278 .\end{array}$ https://doi.org/10.1123/kr.2015-0028

Gould, D., Tuffey, S., Udry, E., \& Loehr, J. 1996. Burnout in competitive junior tennis players: Qualitative content analysis and case studies. The Sport Psychologist, 10, 341-366. https://doi.org/10.1123/tsp.10.4.341

Gould, D., Tuffey, S., Udry,E., \& Loehr, J. 1997. Burnout in competitive junior tennis players; Individual Differences in the burnout experience. The Sport Psychologist, 11, 257-276. https://doi.org/10.1123/tsp.11.3.257

Horton, S. (2012). Environmental influences on early development in sport experts. In Baker, J., Cobley, S. S. \& Schorer, J. (Eds). Talent Identification and Development in Sport: International Perspectives: New York: Routledge.

Hulteen, R.M., Morgan, P., Barnett, L.M., Stodden, D.F. \& Lubans, D.R. (2018). Development of Foundational Movement Skills: A Conceptual Model for Physical Activity Across the Lifespan. Sports Medicine. https://doi.org/10.1007/s40279-018-0892-6

International Physical Literacy Association (2016). Definition of Physical Literacy. https://www.physical-literacy.org.uk/.

Jayanthi, N., Dechert, A., Durazo, R., Luke, A. (2011). Training and specialization risks in junior elite tennis players. Journal of Medicine and Science in Tennis. 16:14-20.

Jayanthi, N., Pinkham, C., Dugas, L., Patrick, B. \& Labella, C. (2013). Sports specialization in young athletes: evidencebased recommendations. Sports Health. 5:251-257. https://doi.org/10.1177/1941738112464626

Jayanthi, N.A., LaBella, C.R., Fischer, D., Pasulka, J. \& Dugas, L.R. (2015). Sports-specialized intensive training and the risk of injury in young athletes: a clinical case-control study. American Journal of Sports Medicine. 43:794-801. https://doi.org/10.1177/0363546514567298

Jayanthi, N.A. \& Dugas, L.R. (2017). The Risks of Sports Specialization in the Adolescent Female Athlete. Strength and Conditioning Journal. 20-26. https://doi.org/10.1519/SSC.0000000000000293

Jayanthi, N.A., Holt, D.B., LaBella, C.R. \& Dugas, L.R. (2018). Socioeconomic Factors for Sports Specialization and Injury in Youth Athletes. Sports Health. (In Press). https://doi.org/10.1177/1941738118778510

LaPrade, R.F., Agel, J., Baker, J., Brenner, J.S., Cordasco, F.A., Coté, J., Engebretsen, L., Feeley, B.T., Gould, D., Hainline, B., Hewett, T.,
Jayanthi, N., Kocher, M.S., Myer, G.D., Nissen, C.W., Philippon, M.J. \& Provencher M.T. (2016). AOSSM early sport specialization consensus statement. Orthopaedic Journal of Sports Medicine. 4(4): 2325967116644241.

https://doi.org/10.1177/2325967116644241

Miller, S.M. \& Siegel, J.T. (2017). Youth sports and physical activity: The relationship between perceptions of childhood sport experience and adult exercise behavior. Psychology of Sport and Exercise. 33, 85-92. https://doi.org/10.1016/j.psychsport.2017.08.009

Pasulka, J., Jayanthi, N., McCann, A., Dugas, L.R. \& LaBella, C. (2017). Specialization patterns across various youth sports and relationship to injury risk. The Physician and Sportsmedicine. https://doi.org/10.1080/00913847.2017.1313077

Roetert, E.P. \& Bales, J. (2014). A Global Approach to Advancing the Profession of Coaching. International Sport Coaching Journal., 1, 2-4. https://doi.org/10.1123/iscj.2013-0026

Roetert, E.P., Kovacs, M., Crespo, M. \& Miley, D. (2016). The role of tennis in developing physical literacy. ITF Coaching and Sport Science $\begin{array}{llllll}\text { Review. } & 69 & \text { (24): } & 3 & - & 5 .\end{array}$ https://doi.org/10.52383/itfcoaching.v24i69.184

Roetert E.P., Ellenbecker, T.S. \& Kriellaars, D. (2018). Physical Literacy: why should we embrace this construct? British Journal of Sports Medicine. Epub ahead of print: April 13. https://doi.org/10.1136/bjsports-2017-098465

Sieghartsleitner, R., Zuber, C., Zibung, M. \& Conzelmann, A. (2018). "The Early Specialised Bird Catches the Worm!" - A Specialised Sampling Model in the Development of Football Talents. Frontiers in Psychology. 9, 1-12. https://doi.org/10.3389/fpsyg.2018.00188

CONTENIDO ITF ACADEMY RECOMENDADO (HAZ CLICK ABAJO)

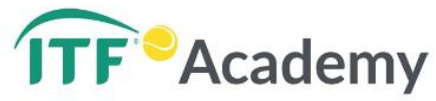

Derechos de Autor (c) 2018 E. Paul Roetert, Ronald B. Woods y Neeru A. Jayanthi

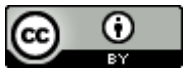

Este texto está protegido por una licencia CreativeCommons 4.0.

Usted es libre para Compartir —copiar y redistribuir el material en cualquier medio o formato- y Adaptar el documento — remezclar, transformar y crear a partir del material- para cualquier propósito,, incluso para fines comerciales, siempre que cumpla la condición de:

Atribución: Usted debe dar crédito a la obra original de manera adecuada, proporcionar un enlace a la licencia, e indicar si se han realizado cambios. Puede hacerlo en cualquier forma razonable, pero no de forma tal que sugiera que tiene el apoyo del licenciante o lo recibe por el uso que hace de la obra. 